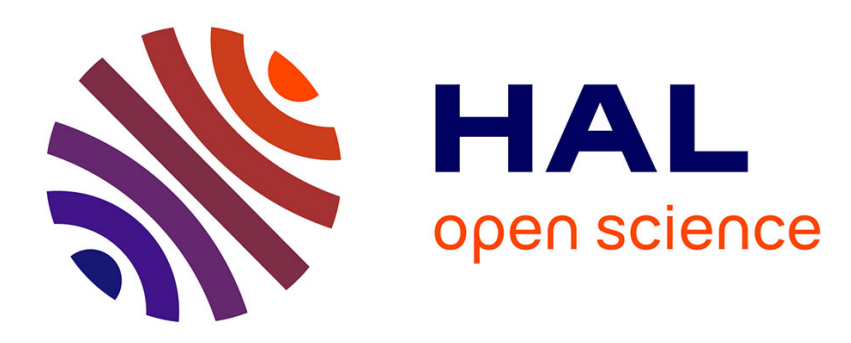

\title{
Appareil pour l'étude des fluides aux pressions élevées par la méthode des impulsions
}

Claude Magnien

\section{To cite this version:}

Claude Magnien. Appareil pour l'étude des fluides aux pressions élevées par la méthode des impulsions. Revue de Physique Appliquée, 1970, 5 (2), pp.263-268. 10.1051/rphysap:0197000502026300 . jpa00243386

\section{HAL Id: jpa-00243386 https://hal.science/jpa-00243386}

Submitted on 1 Jan 1970

HAL is a multi-disciplinary open access archive for the deposit and dissemination of scientific research documents, whether they are published or not. The documents may come from teaching and research institutions in France or abroad, or from public or private research centers.
L'archive ouverte pluridisciplinaire HAL, est destinée au dépôt et à la diffusion de documents scientifiques de niveau recherche, publiés ou non, émanant des établissements d'enseignement et de recherche français ou étrangers, des laboratoires publics ou privés. 


\title{
APPAREIL POUR L'ÉTUde DES FLUIDES AUX PRESSIONS ÉLEVÉES PAR LA MÉTHODE DES IMPULSIONS
}

\author{
par Claude MAGNIEN \\ Laboratoire des Hautes Pressions (C. N. R. S.), 92, Bellevue
}

(Reçu le 23 juin 1969, révisé le 14 octobre 1969)

\begin{abstract}
Résumé. - L'auteur, après un bref rappel de la théorie, et des principales caractéristiques de l'appareil électronique associé, décrit un dispositif comprenant un émetteur et un récepteur d'ultrasons, à parcours variable, placé dans une enceinte étanche jusqu'à des pressions de 1200 bars. Le thermostat contenant l'ensemble permet de maintenir une stabilité de température du centième de degré centigrade entre 25 et $75^{\circ} \mathrm{C}$.

Abstract. - The author, after a brief review of the theory and of the main principles of the associated electronic apparatus, describes an installation having a sender and a receiver of ultrasonic waves, with a variable path, mounted in a vessel able to withstand pressures up to 1200 bars. The thermostat in which the ensemble is placed permits a tempetature stability of one hundredth of a degree between 25 and $75^{\circ} \mathrm{C}$.
\end{abstract}

I. Rappel de la théorie. - L'équation générale d'une onde plane amortie se propageant suivant un axe $\mathrm{OX}$, est de la forme :

$$
A=A_{0} \mathrm{e}^{-\alpha x} \cos \omega\left(t-\frac{x}{a}\right) .
$$

Les différents termes de cette formule sont :

$A$ : amplitude de l'onde à la distance $x$ de l'émetteur ;

$A_{0}$ : amplitude de l'onde à l'origine ;

$\alpha$ : coefficient d'atténuation;

$\omega:$ pulsation de l'onde :

$t$ : temps mis par l'onde pour parcourir la distance $x$;

$a$ : célérité de l'onde.

La méthode des impulsions permet de calculer la célérité et le coefficient d'atténuation. Dans un milieu non dispersif, la célérité $a$ est liée à la densité $\rho$ et au coefficient de compressibilité adiabatique $\chi_{s}$ par la relation :

$$
a^{2}=1 / \rho \chi_{s} .
$$

Le coefficient d'atténuation, dans le cas des fluides denses [I], est lié à la fréquence $f$ de l'onde acoustique et aux coefficients de viscosité de cisaillement $\eta$ et de volume $\eta^{\prime}$ par la relation :

$$
\frac{\alpha}{f^{2}}=\frac{2 \pi^{2}}{\rho a^{3}}\left[\frac{4}{3} \eta+\eta^{\prime}\right] .
$$

Nous avons négligé le terme dû à la conductibilité calorifique. Par exemple, la contribution de cet effet n'est que le millième de celle des coefficients de viscosité, dans nos mesures dans l'azote [II].
II. Principe de l'appareil électronique. - Un générateur [III] envoie 250 impulsions par seconde à l'émetteur piézoélectrique en titanate de baryum (Quartz et Silice, qualité $\mathrm{T} 57$ ) de $5,18 \mathrm{MHz}$ de fréquence fondamentale. A chaque impulsion reçue, celui-ci vibre et envoie une impulsion acoustique dans le milieu étudié. Face à l'émetteur, à une distance $l$, le récepteur se met à vibrer mécaniquement au temps :

$$
t=\frac{l}{a}
$$

Cette vibration engendre une tension électrique entre les armatures du récepteur. Celui-ci réfléchit l'impulsion acoustique qui repart vers l'émetteur jouant alors le rôle de réflecteur. Ce processus se poursuit jusqu'à l'atténuation complète de l'onde. Les impulsions électriques, proportionnelles aux impulsions acoustiques, apparaissant aux bornes du récepteur, sont amplifiées par des étages électroniques. Elles sont traitées ensuite selon deux procédés.

A. Une chaine digitale met ces impulsions sous forme de tops, en impulsions normalisées, de 8 volts d'amplitude et de 10 nanosecondes par volt de temps de montée. Une porte électronique laisse passer le top d'émission et supprime un nombre quelconque de tops d'échos. Nous ne conservons généralement que le top le plus éloigné. Ce procédé permet d'obtenir la célérité avec une grande précision. En effet, de la relation :

$$
a=\frac{n l}{t}
$$

où $n$ est le nombre de trajets et $t$ le temps mesuré par 
un chronographe électronique [A 1195 - Rochar $\Delta t=25 \mathrm{~ns}$ ], nous déduisons l'erreur relative :

$$
\frac{\Delta a}{a}=\frac{\Delta n}{n}+\frac{\Delta l}{l}+\frac{\Delta t}{t} .
$$

En remarquant que :

1) $\Delta n / n=0$ car $n$ est un entier. Une erreur de comptage conduirait à une valeur aberrante de la célérité, facile à remarquer et à corriger.

2) $\Delta t / t=50 \mathrm{~ns} / t$.

Les 50 ns sont la somme des erreurs de 25 ns commises au départ et à l'arrêt du comptage. Le milieu étudié se comporte comme un filtre passe-bas. Le temps de montée du flanc avant de l'onde acoustique augmente donc à chaque traversée de ce filtre. Mais, d'une part, le seuil de déclenchement de mise en forme des signaux étant voisin de zéro et, d'autre part, la sensibilité du chronographe étant de $25 \mathrm{~ns}$, l'erreur due à la déformation de l'onde n'a pu être décelée. Elle est de l'ordre de la nanoseconde par trajet.

Le seuil de déclenchement du comptage est réglé à 1 volt. Les impulsions normalisées ont donc un temps de montée de 10 ns. Une imprécision de lecture de 1 digit, c'est-à-dire $25 \mathrm{~ns}$, commencerait à apparaître seulement pour un temps de montée supérieur à $25 \mathrm{~ns}$. Il n'y a donc pas d'erreur due à la mise en forme des échos.

Finalement l'erreur de 50 ns que nous considérons dans nos calculs provient uniquement de l'absence de synchronisation entre l'horloge pilote du chronographe et le générateur d'impulsions ultrasonores.

3) $\Delta l / l$ se calcule par étalonnage de l'appareil.

Méthode à parcours fixe. - Nous avons employé d'abord ce procédé simple, à pression atmosphérique, avant de concevoir l'appareil décrit dans le présent article.

L'appareil à parcours fixe est en invar. En maintenant la température et la pression constantes pendant les mesures, la célérité peut être considérée comme une constante et l'erreur relative de celle-ci est nulle. Alors l'erreur relative sur la longueur est égale à celle du temps

$$
\frac{\Delta l}{l}=\frac{\Delta t}{t}
$$

L'erreur relative sur la célérité devient :

$$
\frac{\Delta a}{a}=2 \frac{\Delta t}{t}=\frac{100}{t \times 1.000}
$$

La longueur du parcours direct se calcule à partir d'une mesure du temps nécessaire à une impulsion pour faire $n$ allers et retours et de la célérité d'un liquide étalon tel que l'eau distillée.

A titre d'exemple, nous avons obtenu pour un trajet direct de $10 \mathrm{~cm}$, dans l'eau distillée, un temps de
2500 microsecondes, correspondant à 37 trajets. L'erreur relative était de :

$$
\frac{\Delta a}{a}=\frac{100}{25 \times 10^{5}}=4 \times 10^{-5} .
$$

Afin de comparer nos résultats à ceux d'autres spécialistes, nous avons repris le tableau publié par W. D. Wilson [IV] en y ajoutant le résultat de nos mesures, faites dans l'eau distillée, à $30^{\circ} \mathrm{C}$ et à pression atmosphérique.

\begin{tabular}{lcc}
\multicolumn{1}{c}{ Auteurs } & Date & en $\mathrm{m} \cdot \mathrm{s}^{-1}$ \\
Matthews & - & - \\
Kuwahara & 1939 & 1504,4 \\
Del Grosso & 1939 & 1504,4 \\
Greenspan & 1952 & 1509,6 \\
Wilson & 1958 & 1509,44 \\
Magnien & 1959 & 1509,66 \\
& 1965 & 1509,54
\end{tabular}

Méthode à parcours variable. - L'appareil que nous décrirons permet de faire varier la distance entre l'émetteur et le récepteur au moyen d'une vis micrométrique à raison de un millimètre par tour, à $1,2 \times 10^{-3} \mathrm{~mm}$ près. La vis a été contrôlée et cette imprécision se conserve si nous faisons $m$ tours.

En maintenant la célérité constante $(p=$ Cte, $T=$ Cte), nous mesurons les temps de propagation $t^{\prime}$ et $t^{\prime \prime}$ pour deux trajets $l-m$ et $l$. Le trajet $l$ se déduit de

$$
\begin{gathered}
l=\frac{m t^{\prime \prime}}{t^{\prime \prime}-t^{\prime}} \\
\frac{\Delta l}{l}=\frac{\Delta m}{m}+\frac{\Delta t^{\prime \prime}}{t^{\prime \prime}}+\frac{\Delta\left(t^{\prime \prime}-t^{\prime}\right)}{t^{\prime \prime}-t^{\prime}}
\end{gathered}
$$

En prenant soin de considérer les temps correspondants à un même nombre d'échos $n$, les temps s'expriment en fonction de $n$ et de $m$.

$$
t^{\prime \prime}=n \frac{l}{a} \quad t^{\prime}=n \frac{l-m}{a} .
$$

Nous devons exprimer ces temps en nanosecondes dans la relation qui donne l'erreur relative

$$
\frac{\Delta l}{l}=\frac{\Delta m}{m}+\frac{1}{1000} \frac{a}{n}\left(\frac{50}{l}+\frac{100}{m}\right) \text {. }
$$

Nous constatons que $\Delta l / l$ décroît si $m$ et $n$ augmentent. Le nombre d'échos $n$ augmente si le fluide étudié est peu absorbant et le déplacement $m$ est limité par la zone de Fresnel. Nous devons donc mentionner que la précision sur les mesures de célérité dépend non seulement de l'appareil mais aussi du coefficient d'absorption acoustique du fluide étudié.

Il est à remarquer que le temps réel est toujours supérieur au temps enregistré. Ainsi il n'y a pas d'imprécision sur le sens de l'erreur et tous les $\Delta t$ sont positifs dans la relation de $\Delta l / l$. Pour la célérité, 
$t$ étant connu par défaut, $a$ est connu par excès. Nous aurons donc toujours

$$
a_{\text {mesuré }}=a_{\text {réelle }}+K \Delta a
$$

où $K$ est un coefficient entre 0 et 1 .

Résultat de mesures. - Nous avons mesuré la célérité dans l'azote (pureté : 99,998, pression : 1126 bars, température : $25^{\circ} \mathrm{C}$ ). Pour étalonner le parcours, nous avons obtenu 8 échos, correspondant à 17 trajets $(n=17)$.

$$
\begin{aligned}
t^{\prime} & =1958,400 \times 10^{3} \mathrm{~ns} \\
t^{\prime \prime} & =2337,100 \times 10^{3} \mathrm{~ns} \\
t^{\prime \prime}-t^{\prime} & =378,700 \times 10^{3} \mathrm{~ns} \\
m & =20 \mathrm{~mm}
\end{aligned}
$$

d'où

$$
l=123,4 \mathrm{~mm} \quad \text { et } \quad a=897,8 \mathrm{~m} \cdot \mathrm{s}^{-1} .
$$

L'erreur relative sur la longueur est ici de :

$$
\frac{\Delta l}{l}=\left(\frac{1,2}{20}+\frac{50}{2337}+\frac{100}{379}\right) 10^{-3}=0,34 \times 10^{-3} .
$$

L'erreur relative sur la célérité par la méthode à parcours variable est alors de :

$$
\frac{\Delta a}{a}=\left(0,34+\frac{50}{2337}\right) 10^{-3}=3,6 \times 10^{-4}
$$

et

$$
\Delta a=0,3 \mathrm{~m} \cdot \mathrm{s}^{-1} .
$$

D’après la remarque, la célérité s'exprime par :

$$
a=897,8=0,3 \mathrm{~m} \cdot \mathrm{s}^{-1} .
$$

En résumé, la méthode des impulsions que nous avons utilisée, à parcours variable, réflexions multiples et suppression d'échos permet une grande précision. Cependant celle-ci est limitée par les dimensions du dispositif mécanique. Il n'autorise qu'un parcours relativement court entre l'émetteur et le récepteur puisqu'il est placé dans une enceinte haute pression. La précision est limitée aussi par le fluide étudié si celui-ci atténue fortement les signaux. Il n'est possible alors que de mesurer un petit nombre d'échos.

B. UNE ChaINE ANAlogiQUe DÉTECTE ET AMPLIFIE LINÉAIREMENT LES ÉCHOS. - Un dispositif de retard permet de déplacer un signal de porte. Les échos sont envoyés à une porte analogique. Lorsqu'un signal de porte chevauche un écho, la porte est débloquée et ne laisse passer que cet écho qui est envoyé à un voltmètre de crête. Celui-ci donne une tension continue proportionnelle à l'amplitude de l'écho. Nous pouvons ainsi mesurer aisément avec un voltmètre continu l'amplitude de chaque écho afin de calculer le coefficient d'absorption. Grâce à notre dispositif à parcours variable, nous avons utilisé trois méthodes :

1) Méthode des deux parcours ;

2) Méthode des multiples parcours ;
3) Méthode du signal direct.

Cette dernière s'est révélée la plus précise. Elle consiste à mesurer l'amplitude $A_{1}$ du premier signal capté par le récepteur situé à une distance minimale $l_{0}$ de l'émetteur, puis à faire varier le parcours, de centimètre en centimètre, par exemple, jusqu'à la distance maximale $l_{M}$. En traçant la courbe des amplitudes en fonction de la distance, nous obtenons une exponentielle, ce que nous vérifions en traçant la courbe obtenue par les logarithmes des amplitudes. Nous devons obtenir une droite si les mesures ont été faites dans la zone de Fraunhofer. Les longueurs étant exprimées en centimètres, le coefficient d'absorption a pour valeur :

$$
\alpha=\frac{20}{2,3\left(l_{M}-l_{o}\right)} \log _{10} \frac{A_{o}}{A_{M}} \text { en db.cm }{ }^{-1} .
$$

Il est nécessaire de tracer les courbes pour s'assurer que les mesures sont faites dans la zone de Fraunhofer. Cette zone ne peut être calculée aisément, car un émetteur piézoélect. ique ne vibre pas comme un piston [V]

L'erreur relative du coefficient d'absorption s'exprime par :

$$
\begin{aligned}
\frac{\Delta \alpha}{\alpha} & =\frac{\Delta\left(\log A^{\prime} / A\right)}{\log A^{\prime} / A}+\frac{\Delta\left(l-l^{\prime}\right)}{l-l^{\prime}} \\
& \simeq \frac{2 E}{\log A^{\prime} / A}+\frac{2 \Delta l}{l-l^{\prime}}
\end{aligned}
$$

$E$ est l'erreur relative commise sur la mesure des tensions $\left(E=2 \times 10^{-2}\right.$ avec le voltmètre analogique utilisé). Lors des mesures d'atténuation, la différence de parcours $l-l^{\prime}$ vaut $12 \mathrm{~cm}$ et $\Delta l$ vaut $1,2 \times 10^{-4} \mathrm{~cm}$. En exprimant le logarithme népérien en fonction de $\alpha$ en $\mathrm{db} / \mathrm{cm}$ et en tenant compte de la valeur de $l-l^{\prime}$, nous obtenons :

$$
\log \frac{A^{\prime}}{A}=1,38 \alpha \quad \text { avec } \quad \alpha \text { en db.cm }{ }^{-1}
$$

et

$$
\frac{\Delta \alpha}{\alpha}=\frac{2 E}{1,38}+2 \times 10^{-5}
$$

En examinant le premier terme, nous voyons que l'erreur relative est grande, croît si $\alpha$ est faible, ce qui est le cas ici puisque $\alpha$ est exprimé en $\mathrm{db}$, valeur souvent inférieure à l'unité. $\alpha$ est supérieur à l'unité. Ce cas se présente si le fluide est très absorbant ou si le trajet $l-l^{\prime}$ est important. Cette condition impose l'emploi d'une bombe plus longue que celle décrite ici.

La méthode des impulsions englobe, en fait, deux types de signaux acoustiques. Ce sont, soit des trains d'oscillations, soit de simples impulsions. Nous avons employé ce second type d'impulsions.

Nous avons réalisé un appareil permettant de mesurer la célérité et l'atténuation des ultrasons dans les fluides comprimés. A notre connaissance, il existe peu d'appareils permettant d'obtenir des parcours variables sous hautes pressions. R. Bass [VI] est monté à 
100 atmosphères, en reprenant l'étude d'appareils conçus pour 20 atmosphères par Sittig. G. Holton nous a signalé un autre appareil utilisé par Litovitz.

Notre appareil comprend :

1) la tête de mesure, contenant les transducteurs piézoélectriques et le mécanisme de déplacement,

2) l'enceinte haute pression, ou bombe, dans laquelle se place la tête de mesure,

3) le thermostat.

III. Tête de mesure. - Chaque pastille piézoélectrique, en titanate de baryum, a subi une argenture spéciale (Fig. 1). Les pastilles sont fixées, de façon identique, à un support cylindrique par un collage à l'araldite imprégnée de poudre d'argent. Le montage est réalisé de telle sorte que les ondes acoustiques se réfléchissent ainsi sur une paroi plane.

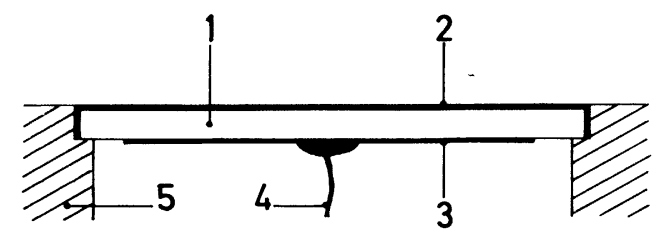

Fig. 1. - Montage des pastilles piézoélectriques : 1. Titanate de baryum (diamètre $15 \mathrm{~mm}$, épaisseur $0,5 \mathrm{~mm}$ ); 2. Dépôt d'argent sur la face d'émission ; 3 . Dépôt d'argent sur la face arrière; 4. Soudure du conducteur électrique; 5. Support cylindrique.

La tête de mesure (Fig. 2) comprend essentiellement l'émetteur (1) fixé à une extrémité d'un tube (2) dans lequel coulisse le récepteur (3). Toutes les pièces sont en acier inoxydable, à l'exception du tube (2) qui est en girobronze. Le support de l'émetteur est solidaire d'un ensemble mécanique qui permet de régler le parallélisme des faces des transducteurs. Le support du récepteur est fixé à une pièce cylindrique dont la face arrière est vissée sur une tige filetée (6) qui lui transmet le mouvement de translation. Les faces de chaque pastille sont en contact avec le fluide étudié. Elles sont donc chargées par la même impédance acoustique et l'énergie émise par le transducteur se scinde en deux parties égales. Pour dissiper l'énergie de l'onde arrière, nous avons utilisé une grille en feuille d'argent froissée placée dans la cavité arrière.

Le mécanisme de déplacement permet de faire varier la distance entre les transducteurs. Un mouvement de rotation extérieur est transformé en un mouvement de translation intérieur et le déplacement intérieur se fait à volume constant. Une vis pointeau (8) engagée dans une rainure longitudinale (7) de la tige filetée (6) n'autorise à celle-ci qu'un mouvement de translation lorsque la pièce (4) tourne. Deux tubes de guidage (2) permettent d'obtenir 124 ou $248 \mathrm{~mm}$ de parcours utile maximal.

L'étanchéité est faite par l'ensemble (10), suivant le

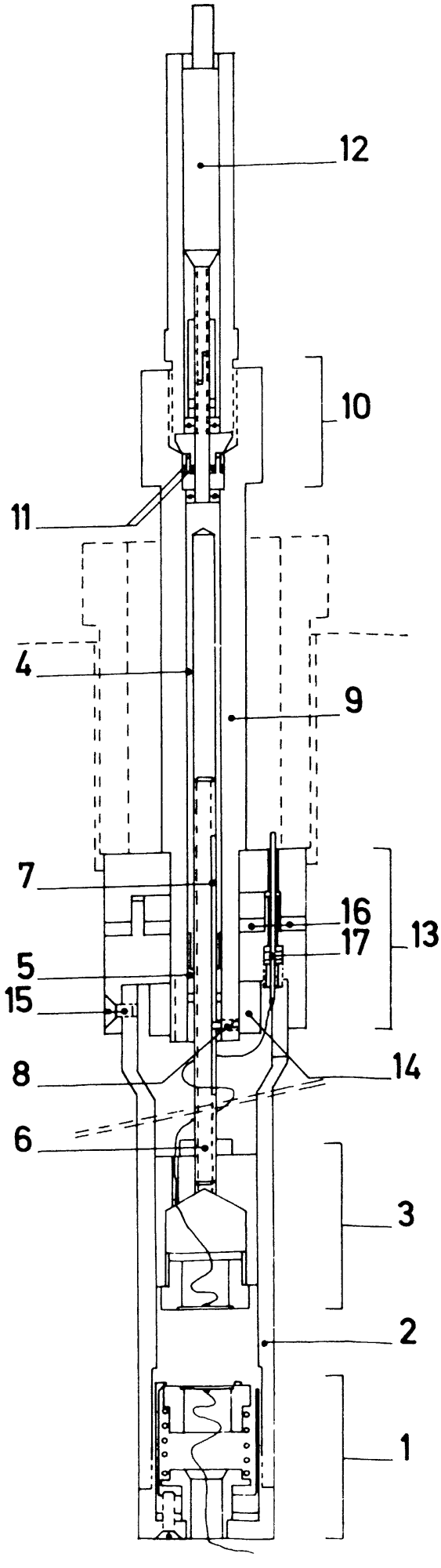

FIG. 2. - Tête de mesure : 1. Ensemble d'émission; 2. Tube de guidage ; 3 . Ensemble de réception; 4. Tige transmettant la rotation; 5. Embout fileté brasé à la tige $(4) ; 6$. Tige filetée; 7. Rainure pour interdire la rotation de la tige $(6) ; 8$. Vis pointeau de blocage engagée dans la rainure (7); 9 . Corps de mouvement ; 10. Ensemble d'étanchéité ; 11 . Joints en téflon; 12. Axe de commande extérieure; 13. Ensemble d'étanchéité bombe-tête de mesure; 14 . Ecrou de blocage; 15 . Vis fixant le tube (2) à l'ensemble (13); 16. Joints d'étanchéité en viton; 17. Passage de courant. 
principe des joints auto-serreurs à aire non supportée. Les joints (11) sont en téflon. La tige renforcée (12) transmet de l'extérieur le mouvement de rotation à la pièce (4). La pièce (9) contenant le mécanisme de déplacement est fixée à l'ensemble d'étanchéité (13) de la bombe par un écrou (14). Le tube de guidage (2) est solidarisé à ce même ensemble par trois vis (15).

La tête de mesure comporte donc les transducteurs situés à l'intérieur d'un tube de guidage fixé d'un côté de cet ensemble d'étanchéité et, de l'autre côté de cet ensemble, la pièce contenant le mécanisme de déplacement. Cette tête a une hauteur de $68 \mathrm{~cm}$ avec le grand parcours. Le tube de guidage (2) a $3 \mathrm{~cm}$ de diamètre intérieur et $4 \mathrm{~cm}$ de diamètre extérieur.

IV. Bombe pour hautes pressions. - Le corps de bombe est en acier inoxydable, qualité $\times 13$ traité à 100-110 kg. L'ensemble d'étanchéité est muni de joints auto serreurs à aire non supportée en viton (16). Deux passages coaxiaux de courant (17) et un passage de thermocouple [VII] traversent cet ensemble. La mise au point et l'élimination des fuites de gaz aux pressions élevées ont présenté de nombreuses difficultés. Une modification de l'ensemble d'étanchéité est actuellement étudiée afin d'utiliser les joints toriques. Le corps de bombe a $52 \mathrm{~cm}$ de hauteur et $12 \mathrm{~cm}$ de diamètre. Une cheminée (Fig. 3) en cuivre, évite que la poudre d'aluminium ou éventuellement l'huile employée dans le thermostat pénètre dans les filetages de l'écrou de serrage.

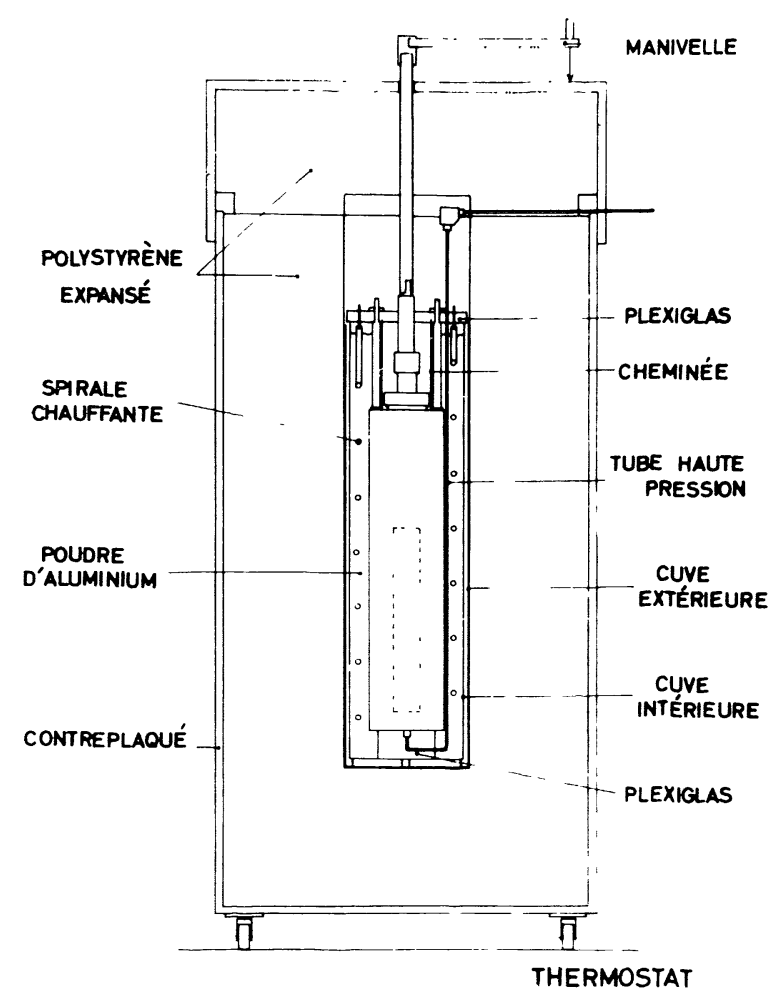

FIG. 3. - Thermostat.
La pression est lue sur un manomètre Heise, gradué de 0 à 2000 bars, permettant de lire la pression à un bar près.

V. Thermostat. - Une spirale chauffante entoure la bombe, et les calories sont transférées à celle-ci par de la poudre d'aluminium. La cheminée est fermée par une plaque de plexiglas, traversée par trois colonnes solidaires de la bombe. Cette dernière est munie, à sa partie inférieure, de deux supports en plexiglas. La bombe, la spirale et la poudre sont dans une cuve en acier. Cet ensemble est introduit, à l'aide d'un palan, dans une enceinte isolante en polystyrène expansé de $60 \mathrm{~cm}$ de côté et de $145 \mathrm{~cm}$ de haut. La spirale chauffante est alimentée en $110 \mathrm{~V}$ alternatif par l'intermédiaire d'un régulateur de température dont l'élément sensible est une thermistance. Après un palier à $75^{\circ} \mathrm{C}$, nous avons arrêté le régulateur. De la chute de température en fonction du temps, nous avons déduit l'inertie thermique moyenne de l'installation. Elle est de $0,92{ }^{\circ} \mathrm{C}$ par heure.

Pour mesurer les températures, nous avons placé un thermocouple contre la paroi de la bombe, dans la poudre d'aluminium, et un thermocouple à l'intérieur de la bombe. En cours d'expérience, lorsque les températures des deux thermocouples sont égales, nous considérons le système en équilibre thermique et nous procédons à une série de mesures.

Ce thermostat nous a permis d'obtenir une stabilité de température de un centième de degré centigrade.

Conclusion. - Notre installation a permis de mesurer la célérité et l'atténuation des ultrasons dans les fluides sous hautes pressions par la méthode des impulsions avec parcours variable, alors que, généralement, ces mesures simultanées sont faites à basses pressions par la méthode interférométrique (parcours et fréquences variables) ou à hautes pressions par la méthode des impulsions (avec deux parcours fixes). Le dispositif mécanique de variation du parcours s'est montré d'un emploi très commode. Les techniques électroniques employées nous ont permis de mesurer l'atténuation avec une précision acceptable et la célérité avec une très grande précision. Ces précisions n'ont été significatives que grâce à l'utilisation d'un thermostat donnant le centième de degré et d'un manomètre fidèle et précis au bar près.

Nous avons mesuré la célérité dans l'argon et l'azote entre 100 et 1200 bars, à 25,50 et $75^{\circ} \mathrm{C}$ avec des précisions relatives variant entre 3,5 et 6 dix millièmes. Les résultats détaillés de ces mesures feront l'objet d'un prochain article [II]. L'atténuation n'a pu être mesurée qu'à 25 et $50^{\circ} \mathrm{C}$. A $75^{\circ} \mathrm{C}$, les valeurs obtenues étaient trop dispersées. Nous avons l'intention d'étudier ces gaz à cette température, puis d'entreprendre l'étude de plusieurs autres gaz afin de provoquer, par les hautes pressions, des processus de relaxation structurelle. 


\section{Bibliographie}

[I] Litovitz (T. A.), ( Effet de la pression sur la relaxation ultrasonore dans les liquides », Propriétés optiques et acoustiques des fluides, Ed. C. N. R. S., Paris, 1959, 419-435.

[II] Magnien (C.), "Célérité et atténuation des ultrasons dans l'azote et l'argon entre 100 et 1200 bars à 25,50 et $75^{\circ} \mathrm{C}$ ) (à paraître).

[III] MAGNIEN (C.), "Générateur-récepteur d'ultrasons pour l'étude des fluides aux pressions élevées par la méthode des impulsions ) (à paraître).

[IV] WILson (W. D.), " Tables for the speed of sound in distilled water and in sea water ), Navord Report
6747 (nov. 1959), U. S. Naval Ordnance Laboratory White Oak, Silver Spring, Md, U. S. A

[V] Pajewski (W.), Cazanhes (C.), Garnier (J. L.), "Champ ultrasonore des disques de titanate de baryum et de quartz émettant dans un liquide "), C. R. Acad. Sci., Paris, 1966, 262, 232-234.

[VI] Andreae (J. H.), Bass (R.), Heasell (E. L.) and LAMB (J.), (C Pulse techniques for measuring ultrasonic absorption in liquids ), Acustica, 1958, 8. 131-142.

[VII] Magnien (C.) et Bienaimé (R.), " Passages de courant pour hautes pressions ), Jour. Phys. App., $1968,3,283-285$. 